\title{
A Rare Case of Paediatric Pleomorphic Adenoma of the Parotid Gland
}

Tantry D and Chitra G*

Department of ENT, AJ IMS, Mangalore, India

*Correspondling author: Gowda Chithra, Department of ENT, AJ IMS, Mangalore, India

Received: J une 28, 2021; Accepted: J uly 14, 2021;

Published: July 21, 2021

\begin{abstract}
Pleomorphic adenomas are benign salivary gland tumours, which predominantly affect the superficial lobe of the parotid gland. The "pleomorphic" nature of the tumour can be explained on the basis of its epithelial and connective tissue origin. The tumour has a female predilection between 30-50 years of age. Slowly progressing asymptomatic swelling is the usual presentation of the tumour. Surgical excision of the tumour mass forms the mainstay of treatment, with utmost care taken to preserve the facial nerve.
\end{abstract}

This case report aims to throw light on an interesting case of pleomorphic adenoma of the parotid gland in a 10 years old female patient. The patient presented with a slowly progressing asymptomatic swelling on the right side of the face, which was diagnosed to be pleomorphic adenoma of right parotid gland.

The aim of the study is to discuss how to proceed regarding the further management.

Keywords: Pleomorphic adenoma of parotid; Salivary gland tumours; Parotidectomy

\section{Introduction}

Pleomorphic Adenoma (PA), also known as benign mixed tumour, is the most common salivary tumour, constituting up to two-thirds of all salivary gland neoplasms. Mostly, PA is located in the parotid glands $(85 \%)$, minor salivary glands (10\%), and the submandibular glands (5\%) [1]. In the majority of cases, tumours originate in the superficial lobe. However, occasional cases may involve the deep lobe of the parotid gland and the parapharyngeal space. Minor salivary gland tumours are frequently encountered on the palate, followed by the lip, cheek, tongue and floor of the mouth [2,3]. PA usually manifest as a slow progressing asymptomatic, parotid gland swelling without facial nerve involvement. They are best treated by a wide local excision with good safety margins and follow-up for at least 3-4 years [4]. Salivary gland carcinomas are exceedingly rare in the paediatric population. Their annual incidence is estimated to be around one case per million. The majority of paediatric tumours of the salivary gland are benign, with pleomorphic adenoma being the most common type [5-8]. There is a paucity of clinical and biological details about paediatric salivary gland tumours and their clinical behaviour in the literature. We present a rare case of pleomorphic adenoma of the parotid gland in a 10-year-old child.

\section{Case Presentation}

A 10 years old female patient reported to the Outpatient Department with a chief complaint of slow growing, painless swelling below the right ear since 3 years. The swelling was initially small in size and had progressively increased with time to attain the present size. Past medical and surgical history was non contributory. Extraoral clinical examination revealed a mild facial asymmetry. A welldefined, ovoid, solitary swelling, $3 \times 2 \mathrm{~cm}$ in diameter was seen below the right ear. The swelling had an extent, superiorly $\sim 0.5 \mathrm{~cm}$ from the ear lobe, inferiorly $\sim 0.5 \mathrm{~cm}$ from the angle of the mandible, posterior extent $\sim 2.5 \mathrm{~cm}$ from the mastoid process and anteriorly $\sim 5 \mathrm{~cm}$ from the angle of the mouth. The right ear lobule was slightly everted (Figure 1).

On palpation, the inspector findings were confirmed. A Solitary swelling of about $3 \times 2 \mathrm{~cm}$ noted over the right parotid region. Skin over the swelling is pinchable, firm in consistency, mobile and non-tender. It was not fixed to the underlying structures and the overlying skin. Facial and eye movements were normal on examination. Intraoral clinical examination was unremarkable. A provisional diagnosis of benign tumour of the left parotid gland was taken into consideration. Pleomorphic adenoma, Warthin's tumour and neuroma of the facial nerve (nerve sheath tumour) were considered as the most probable differential diagnosis. Warthin's tumour is usually seen in elderly males with a history of smoking, does not cause eversion of the ear lobe, located in the lower portion of the parotid (near angle of mandible), and 10-15\% cases show bilateral involvement. Benign
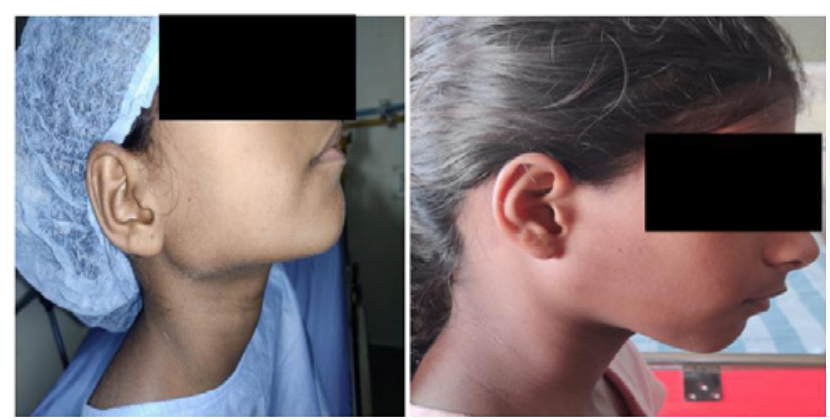

Figure 1: 


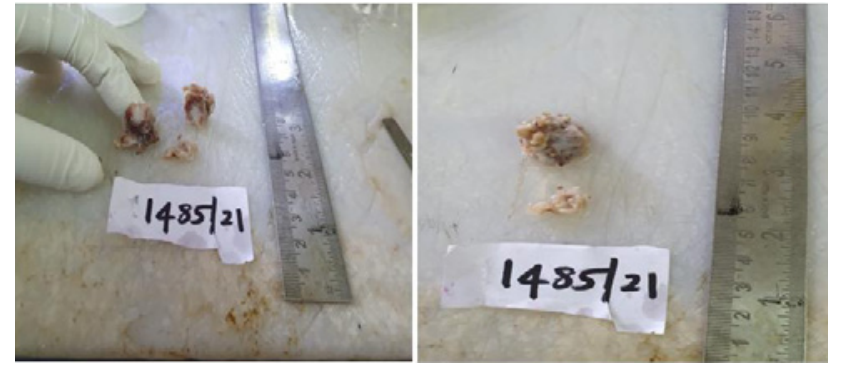

Figure 2:

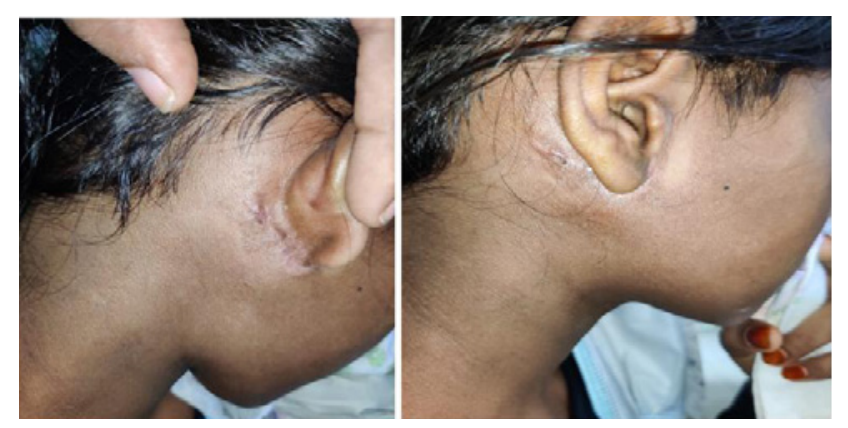

Figure 3:

tumour of nerve sheath origin in the parotid gland poses difficulty in preoperative diagnosis because of the low frequency of occurrence (0.2\% to $1.5 \%)$ (Figure 2$)$.

On palpation, the inspector findings were confirmed. A Solitary swelling of about $3 \times 2 \mathrm{~cm}$ noted over the right parotid region. Skin over the swelling is pinchable, firm in consistency, mobile and non-tender. It was not fixed to the underlying structures and the overlying skin. Facial and eye movements were normal on examination. Intraoral clinical examination was unremarkable. A provisional diagnosis of benign tumour of the left parotid gland was taken into consideration. Pleomorphic adenoma, Warthin's tumour and neuroma of the facial nerve (nerve sheath tumour) were considered as the most probable differential diagnosis. Warthin's tumour is usually seen in elderly males with a history of smoking, does not cause eversion of the ear lobe, located in the lower portion of the parotid (near angle of mandible), and $10-15 \%$ cases show bilateral involvement. Benign tumour of nerve sheath origin in the parotid gland poses difficulty in preoperative diagnosis because of the low frequency of occurrence $(0.2 \%$ to $1.5 \%)$.

The excised tumour mass was $2.1 \times 1 \times 2 \mathrm{~cm}$ in dimension. Histopathology shows an unencapsulated tumour comprising of epithelial and mesenchymal component. The epithelial cells are arranged in sheets, cords, trabeculae pattern and they comprise of plasmacytoid cell with monomorphic vesicular nuclei, and inconspicuous nucleoli admixed with ductal epithelial cells. The stroma is composed of chondromyxoid areas. Adjacent areas shows lobules of serous salivary gland acini. Areas of skeletal muscle and adipocytes noted. The characteristic features of chondromyxoid foci and glandulo - ductal differentiation confirmed the diagnosis of pleomorphic adenoma of the right parotid gland (Figure 3 ).

\section{Differential diagnosis}

Considering the paediatric age group, the possible differential diagnosis include Neurofibroma, Rhabdomyosarcoma, lipoma, dermoid cyst, foreign body reaction, mucocele, buccal space abscess, adenoid cystic carcinoma.

\section{How to proceed:}

To narrow down a diagnosis, further investigations are to be done, which may be invasive or non-invasive. Non-invasive investigations include ultrasound or CT/MRI, which reveals if the swelling is solid/ cystic, hypo/hyper dense, if it is extending to involve the adjacent structures or spaces.

Invasive procedure includes FNAC. Histological examination is the safest way to confirm the diagnosis and for the appropriate treatment.

The histopathology presents varied morphological patterns, showing epithelial and myoepithelial cells with interspersed areas of mesenchymal differentiation. Epithelial cells typically form duct-like structures associated with non-ductal cells presenting varying shapes and forms. Myxoid, cartilaginous, hyaline, or osseous differentiation is appreciated in the stromal component. The stroma is presented as a mixture of gland-like epithelium and mesenchyma-like tissue in varying proportions.

\section{Discussion}

World Health Organization (1972) defined PA as a well-defined tumour characterized by its pleomorphic or mixed appearance. There is intermixing of the clearly recognizable epithelial component with mucoid, myxoid and chondroid component. Although the lesion presents several histological features due to the different compounds with a myxoid or chondroid matrix, it is generally considered a benign neoplasm [9].

The exact etiology is obscure although the incidence increases from 15-20 years after exposure to radiation. Few studies have suggested an association of the tumour with simian virus 40 (SV 40).

Pleomorphic adenomas are generally discovered during routine physical examination, as an asymptomatic mass. PA has a glandular origin in the head and neck region and usually manifests as a mobile, slow progressing, asymptomatic firm swelling that does not cause ulceration of the overlying mucosa [10]. The majority of these tumours measure $2-6 \mathrm{~cm}$ in size when excised. However, large tumour may be seen as a single, irregular nodular mass stretching the overlying skin or mucosa. The tumour may weigh from several grams to more than 8 kilograms. Parotid gland PA is usually seen below the lobule of the ear and overlying the angle of the mandible. Facial nerve weakness is an infrequent sign in parotid tumours although large neglected tumours may present with facial nerve weakness. Oral retro-tonsillar mass/ parapharyngeal space tumour may be a presenting sign in cases of deep lobe involvement [11].

Our patient was a 10 years old female who presented with a slowly enlarging, solitary, asymptomatic swelling on the right side of the face since 3 years. The ocular and facial movements were normal, suggestive of intact facial nerve functions. Grossly, the excised tumour mass measured $2.1 \times 1 \times 2 \mathrm{~cm}$ in diameter. 
Imaging modalities such as Computed Tomography (CT) and Magnetic Resonance Imaging (MRI) are essential aids in diagnosis. MRI is favoured on the basis of better soft tissue delineation, detailed tumour margin description and the tumour relationship with the surrounding structures [12].

MRI findings in the present case were suggestive of a heterogeneous lesion, which appeared hypointense on T1 weighted MR image and hyperintense on T2 weighted MR images. Ultrasound imaging helps to differentiate cystic lesions from solid parotid masses, and is used for assessment of intra-capsular versus extra-capsular tumours. PA's have been identified by ultrasound based on their distinct margins and polycyclic shape.

Ultrasonographic findings of the present case showed a hypoechoic area in the left parotid gland. FNAC is a reliable procedure that can guide the surgeon to choose the right surgical approach. The procedure is usually performed following diagnostic imaging to rule out a vascular lesion although it is not the first choice diagnostic tool [13].

The present case showed a combined pattern of epithelial, myo-epithelial and mesenchymal components on FNAC. The histopathology presents varied morphological patterns, showing epithelial and myoepithelial cells with interspersed areas of mesenchymal differentiation. Epithelial cells typically form duct-like structures associated with non-ductal cells presenting varying shapes and forms. Myxoid, cartilaginous, hyaline, or osseous differentiation is appreciated in the stromal component. The stroma is presented as a mixture of gland-like epithelium and mesenchyma-like tissue in varying proportions.

As pleomorphic adenoma exhibits a varied histopathologic presentation, it may be confused histo-pathologically with myoepithelioma, Adenoid cystic carcinoma, mucoepidermoid carcinoma and basal cell adenoma.

Myoepitheliomas may be considered as a variant of pleomorphic adenoma, but lacking the typical feature of glandulo ductal differentiation. Another characteristic feature of myothelioma is the absence of chondromyxoid or chondroid foci. Chondromyxoid foci and glanduloductal differentiations are the hallmark feature in pleomorphic adenomas.

Adenoid cystic carcinoma shows epithelial and myoepithelial differentiation in three forms: Cribriform, Tubular, and Solid. However, the infiltrative growth pattern and tendency for perineural invasion are the salient features of adenoid cystic carcinoma.

The intermediary cells in mucoepidermoid carcinoma show similarity to the basal/myoepithelial cells of pleomorphic adenoma. Although the intermediary cells have the potential to produce the extracellular material, they lack the ability to create the myxochondroid stroma. Also, squamous differentiation (when present in pleomorphic adenoma) is generally well developed and may show keratinisation. This feature is less evident in carcinoma.

Basal cell adenoma is a subtype of pleomorphic adenoma, and was previously termed as monomorphic adenoma. The tumour can be histologically differentiated from pleomorphic adenoma by the absence of chondromyxoid stroma and the presence of a uniform basaloid epithelial pattern.

Histopathological features in the current case showed salient features of pleomorphic adenoma. A well-capsulated cellular mass of sheets and islands of epithelial cells and rounded myoepithelial cells, along with myxomatous background and ductal architecture, was clearly evident.

Aggressiveness and extent of the tumour mass and its relation with the facial nerve form the important criteria, which dictate the choice of treatment of pleomorphic adenoma of the parotid gland. Enucleation, enucleoresection and superficial or total parotidectomy with preservation of the facial nerve formed the mainstay of surgical treatment.

In the present case, superior lobe of the left parotid gland with the tumour mass was excised with utmost care to preserve the facial nerve branches. Pleomorphic adenomas need to be managed diligently as they have a tendency for recurrence and malignant transformation. Rupture of the capsule and subsequent tumour spillage during excision are attributable risk factors for recurrence. Up to $10 \%$ cases show malignant transformation and features predictive of malignant change include advancing age, massive tumour size, a long duration of the mass, occurrence in submandibular salivary gland, and hyalinized connective tissue.

\section{Conclusion}

Salivary gland neoplasms can occur at any site where salivary tissue is present. Pleomorphic adenoma is the commonest salivary gland tumour characterized by diverse histo-morphological features. Early diagnosis and treatment plan entails thorough history taking, clinical examination, coupled with radiographic and histopathological findings. To conclude, juvenile pleomorphic adenoma of the cheek is a rare neoplasm and therefore its diagnosis requires a high index of suspicion. Complete wide surgical excision is the treatment of choice. Recurrence after many years of surgical excision as well as malignant transformation should be a concern and therefore long- term followup is necessary.

\section{References}

1. Bentz BG, Hughes CA, Ludemann JP, et al. Masses of the salivary gland region in children. Arch Otolaryngol Head Neck Surg. 2000; 126: 1435-1439.

2. Jagadishkumar K, Anilkumar MG, Krishna Kumar HC, et al. Pleomorphic adenoma of the cheek in a child: A case report. Dent Res J (Isfahan). 2014; 11: $522-524$.

3. Eveson JW, Cawson RA. Salivary gland tumours. A review of 2410 cases with particular reference to histological types, site, age and sex distribution. J Pathol. 1985; 146: 51-58.

4. Chang EZ, Lee WC. Surgical treatment of salivary gland tumors. J Oral Maxillofac Surg. 1989; 47: 555-558.

5. Camacho AE, Goodman ML, Eavey RD. Pathologic correlation of the unknown solid parotid mass in children. Otolaryngol Head Neck Surg. 1989; 101: 566-571.

6. Orvidas LJ, Kasperbauer JL, Lewis JE, et al. Pediatric parotid masses. Arch Otolaryngol Head Neck Surg. 2000; 126: 177-184.

7. Krolls SO, Trodahl JN, Boyers RC. Salivary gland lesions in children. A survey of 430 cases. Cancer. 1972; 30: 459-469.

8. Ogata H, Ebihara S, Mukai K. Salivary gland neoplasms in children. Jpn J Clin Oncol. 1994; 24: 88-93. 
9. Castro EB, Huvos AG, Strong EW. Tumors of the major salivary glands in children. Cancer. 1972; 29: 312-317.

10. Vaidya AD, Pantvaidya GH, Metgudmath R, Kane SV, D'Cruz AK. Minor salivary gland tumors of the oral cavity: A case series with review of literature. J Cancer Res Ther. 2012; 8: 111-115.

11. Jorge J, Pires R, Alves FA, Perez DE, Kowalski LP, Lopes MA, et al. Juvenile intraoral pleomorphic adenoma: Report of five cases and review of the literature. Int J Oral Maxillofac Surg. 2002; 31: 273-275.
12. Savithri V, Sudha S, Shameena PM, Ipe VV. Juvenile pleomorphic adenoma J Oral Maxillofac Pathol. 2004; 8: 94-95

13. Jain S, Hasan S, Vyas N, Shah N, Dalal S. Pleomorphic adenoma of the parotid gland: Case report of a case with review of literature. Ethiop. J. Health Sci. 2015; 25: 189-194. 